\title{
СОСТОЯНИЕ КОГНИТИВНЫХ ФУНКЦИЙ ПОСЛЕ АНГИОРЕКОНСТРУКТИВНЫХ ОПЕРАЦИЙ НА СОННЫХ АРТЕРИЯХ
}

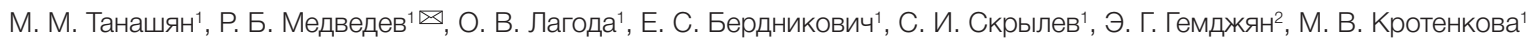

${ }^{1}$ Научный центр неврологии, Москва, Россия

${ }^{2}$ Национальный медицинский исследовательский центр гематологии, Москва, Россия

\begin{abstract}
Атеросклероз сонных артерий является фактором риска ишемического инсульта. Для улучшения мозговой перфузии и предотвращения развития цереброваскулярной патологии и связанных с ней когнитивных нарушений нередко используют хирургическое лечение. Целью данного проспективного поискового исследования было оценить когнитивные функции (КФ) пациентов после операции (открытого или эндоваскулярного вмешательства) на внутренней сонной артерии. В исследование было включено 90 пациентов (средний возраст - 62 года, $71 \%$ мужчин) с атеросклеротическим поражением сонных артерий. КФ оценивали в четырех временных точках (до вмешательства, через 3, 6 и 9 месяцев после него) с использованием когнитивных шкал и измерением когнитивных вызванных потенциалов. Состояние вещества головного мозга до и после вмешательства оценивали по результатам диффузионно-взвешенной магнитно-резонансной томографии (ДВ-МРТ). Через 3 и 6 месяцев после операции у пациентов наблюдали небольшие разнонаправленные изменения КФ (по шкале MMSE), но к концу срока наблюдения (9 месяцам) распределение оценок КФ у пациентов приблизилось к дооперационному ( $p=0,43$ ). Таким образом, ассоциированные с вмешательством (независимо от его вида) изменения КФ носили преимущественно транзиторный характер. Единичные случаи ухудшения (по данным ДВ-МРТ после операции) КФ были ассоциированы с острыми очагами ишемии (как симптомными, так и бессимптомными) в веществе мозга, а также с периоперационным инсультом (1 случай). К факторам риска неблагоприятного прогноза для КФ можно отнести: пожилой возраст и изменения в церебральных артериях. Для оценки связи КФ с множественными интраоперационными эмболиями сосудов мозга требуется более длительное наблюдение.
\end{abstract}

Ключевые слова: сонная артерия, стеноз, стент, эндартерэктомия, эмболия, когнитивные функции, дисперсионный анализ

Финансирование: работа выполнена в рамках государственного задания ФГБНУ НЦН.

Информация о вкладе авторов: М. М. Танашян - разработка дизайна исследования, редактирование рукописи; Р. Б. Медведев — анализ литературы, разработка дизайна исследования, сбор, анализ и интерпретация данных, написание рукописи; О. В. Лагода и Е. С. Бердникович - анализ литературы, разработка дизайна исследования, сбор и интерпретация данных, редактирование рукописи; С. И. Скрылев - проведение ангиохирургических операций, редактирование рукописи; Э. Г. Гемджян - концепция и дизайн исследования, анализ данных, статистический анализ, составление и редактирование рукописи; М. В. Кротенкова - анализ и интерпретация данных изображения, редактирование рукописи.

Соблюдение этических стандартов: исследование одобрено этическим комитетом Научного центра неврологии (протокол № 11/14 от 19 ноября 2014 г.). Все пациенты (или их законно уполномоченные представители) подписали добровольное информированное согласие на проведение оперативного лечения; исследование проведено в соответствии с этическими принципами Хельсинкской декларации 1975 г.

$\bigotimes$ Для корреспонденции: Роман Борисович Медведев

Волоколамское шоссе, д. 80, г. Москва, 125367; medvedev-roman@yandex.ru

Статья получена: 15.08.2019 Статья принята к печати: 29.08.2019 Опубликована онлайн: 16.09.2019

DOI: $10.24075 /$ vrgmu.2019.059

\section{THE STATE OF COGNITIVE FUNCTIONS AFTER ANGIORECONSTRUCTIVE OPERATIONS ON THE CAROTID ARTERIES}

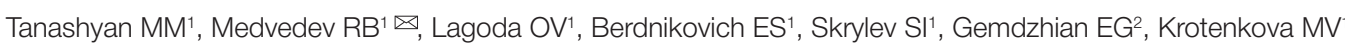

${ }^{1}$ Research Centre of Neurology, Moscow, Russia

${ }^{2}$ National Research Center for Hematology, Moscow, Russia

Carotid artery stenosis is a risk factor for ischemic stroke. Surgical treatment is often used to improve cerebral perfusion and prevent the development of cerebrovascular pathology and related cognitive impairment. The aim of this prospective pilot study was to evaluate the cognitive functions (CF) of patients after surgery (open or endovascular intervention) on the internal carotid artery. The study included 90 patients (mean age 62 years, $71 \%$ of men) with atherosclerotic lesions of the carotid arteries. The CF was evaluated at four time points (before the intervention, 3, 6, and 9 months after) using cognitive scales and measuring cognitive evoked potentials. The state of the brain substance before and after the intervention was evaluated by the results of diffusion-weighted magnetic resonance imaging (DW-MRI). Three and six months after the operation, the patients demonstrated minor and varied CF alterations by the MMSE scale, but by the end of the observation period ( 9 months) the participants had their CF at the level close to that registered before the operation $(p=0.43$ ). Thus, the intervention-associated changes in CF, regardless of the surgical approach, were primarily transient in nature. The rare cases of CF deterioration, as registered by the postoperative DW-MRI scans, were linked to the acute brain ischemia, both symptomatic and asymptomatic, and a perioperative stroke ( 1 case). Advanced age and altered cerebral arteries may be listed as the risk factors for the probable CF deterioration. Evaluation of the connections between CF alterations and multiple cases of intraoperative cerebral vascular embolism requires a longer observation period.

Keywords: carotid artery, stenosis, stent, endarterectomy, emboli, cognitive function, analysis of variance

Funding: the study was ordered by the Research Center of Neurology (Federal Research Institution).

Author contribution: Tanashyan MM — study design development, manuscript editing; Medvedev RB — literature analysis, study design development, data collection, analysis and interpretation, manuscript authoring; Lagoda OV and Berdnikovich ES — literature analysis, study design development, data collection and interpretation, manuscript editing; Skrylev SI — angiosurgery, manuscript editing; Gemdzhian EG — study concept and design, data analysis, statistical analysis, manuscript compilation and editing; Krotenkova MV — image data analysis and interpretation, manuscript editing.

Compliance with ethical standards: the study was approved by the Research Center of Neurology Ethics Committee (protocol № 11/14 of November 19, 2014). All patients or their legally authorized representatives have signed the informed consent for surgery; the study followed the ethical principles of the Declaration of Helsinki (1975).

$\triangle$ Correspondence should be addressed: Roman B. Medvedev

Volokolamskoye shosse, 80, Moscow, 125367; medvedev-roman@yandex.ru

Received: 15.08.2019 Accepted: 29.08.2019 Published online: 16.09.2019

DOI: 10.24075/brsmu.2019.059 
Лидирующие позиции сосудистых заболеваний головного мозга среди причин общей смертности и стойкой утраты трудоспособности населения делают ситуацию с их профилактикой и лечением чрезвычайно актуальной как в социальном, так и медицинском плане [1]. Изменение демографической ситуации в сторону увеличения продолжительности жизни и рост выявляемости сосудистой патологии и связанных с ней когнитивных расстройств требуют дальнейшего изучения сосудистых заболеваний головного мозга.

Когнитивная дисфункция нарушает социальную адаптацию пациентов, приводя не только к ухудшению качества их жизни, но и к снижению адекватного контроля за течением как основного заболевания, так и коморбидных состояний: артериальной гипертензии, атеросклероза, сахарного диабета.

Важнейшей причиной развития ишемического инсульта служит стенозирующее атеросклеротическое поражение брахиоцефальных артерий (БЦА) и особенно внутренней сонной артерии (ВСА). Высокая доля нарушений мозгового кровообращения, развивающихся по атеротромботическому подтипу в общей структуре ишемических инсультов (более 30\%), и значительный процент их возникновения без предшествующих симптомов (80\%) подчеркивают необходимость тщательного обследования пациентов с атеросклерозом.

В патогенезе развития когнитивных нарушений $(\mathrm{KH})$ при атеросклерозе в системе ВСА выделяют два основных механизма - эмболию и церебральную гипоперфузию [2]. При этом независимо от наличия или отсутствия признаков поражения белого вещества, выявляемого при магнитно-резонансной томографии головного мозга, стеноз ВСА является независимым маркером КН. Так, в исследовании, включавшем более 4000 пациентов с асимптомными стенозами ВСА, КН были отмечены в группе больных с выраженной степенью стеноза [3]. Имеются данные о прямой корреляционной связи между увеличением наиболее раннего и информативного маркера атеросклероза - толщиной комплекса интимамедиа ВСА и более низким баллом по результатам нейропсихологического тестирования [4].

Современная доктрина оказания медицинской помощи заключается в изменении фокуса от лечения болезней к активной позиции сохранения своего здоровья каждым человеком. И в этом контексте основной мерой просилактических мероприятий по предотвращению развития цереброваскулярной патологии и КН является обеспечение адекватной перфузии головного мозга.

Наряду с антитромботической терапией большую роль в нормализации мозгового кровотока играют хирургические методы лечения: каротидная эндартерэктомия (КЭЭ) и транслюминальная баллонная ангиопластика со стентированием сонных артерий (КАС) [5-8]. Вместе с несомненным в целом улучшением церебрального кровообращения вследствие коррекции сосудистого русла в настоящее время (с накоплением клинического материала) выявлены и некоторые неблагоприятные моменты. Так, любое оперативное вмешательство (включая ВСА) чревато интраоперационной эмболией и гемодинамической нестабильностью (вплоть до перерыва кровотока) с развитием ишемии мозговой ткани [9]. Согласно данным рандомизированного исследования CREST, частота периоперационного инсульта после КЭЭ и КАС составила 2,3 и 4,1\% соответственно [10]. Результаты дисффузионно-взвешенной магнитно-резонансной томограсии (ДВ-МРТ) свидетельствуют о наличии острых очагов ишемии (ООИ) в веществе мозга у 21\% пациентов, перенесших открытые вмешательства, и у 50\% пациентов, перенесших эндоваскулярные вмешательства [11].

Связь каротидных реваскуляризаций с когнитивными функциями (КФ) остается не до конца ясной из-за наличия многих факторов: 1) гетерогенности пациентов по клиническим проявлениям, локализации и выраженности стеноза, исходного статуса церебральной перфузии, сроках между появлением симптомов и проведением реваскуляризации [12]; 2) используемых видов оценочных нейропсихологических тестов и сроков оценки; 3) вариабельности хирургической техники и критериев классификации постоперационных изменений [13]. Целью нашего исследования было выявить и оценить когнитивные изменения у пациентов, подвергшихся операциям по поводу атеросклеротического поражения ВСА.

\section{ПАЦИЕНТЫ И МЕТОДЫ}

В исследование были включены 90 пациентов (64 мужчин и 26 женщин в возрасте 47-83 лет, средний возраст - 61 год), наблюдавшихся в отделении общей ангионеврологии Научного центра неврологии (Москва). Критерии включения пациентов в исследование: пациенты обоих полов; наличие у пациентов хронических ишемических цереброваскулярных заболеваний (дисциркуляторная энцесралопатия 1-2-й стадии); пациенты, которым в отделении сосудистой и эндоваскулярной хирургии за период с мая 2015 г. по декабрь 2018 г. было выполнено хирургическое лечение ВСА (каротидная эндартерэктомия (КЭЭ) выполнена 27 пациентам, каротидная ангиопластика со стентированием - 63 больным). Критерии исключения: наличие у пациента выраженной сердечной и соматической патологии; тяжелый инсульт; психические нарушения; наличие гемианопсии вследствие нарушения кровообращения в бассейне задней мозговой артерии; наличие исходно выраженных КН (оценка по шкале MMSE < 24 баллов), препятствующих выполнению нейропсихологических тестов.

Диагноз атеросклеротического поражения сонных артерий верифицировали ультразвуковым обследованием на приборе Viamo (Toshiba; Япония) с использованием алгоритма исследования NASCET (North American Symptomatic Carotid Endarterectomy Trial) [14].

Всем пациентам проводили оценку когнитивного статуса до хирургического лечения и затем через 3, 6 и 9 месяцев после него. В исследовании применяли следующие методы оценки КФ: краткую шкалу оценки психического статуса MMSE (Mini-Mental State Examination) [15]; тесты по запоминанию 10 слов, оценки лобной дисфункции (БТЛД), рисования часов, повторения цифр по методу Векслера, концептуализации, а также пробу Шульте и динамический праксис [16]. Выбор методов оценки КФ определяли их функциональной сопоставимостью с исследованием когнитивных вызванных потенциалов (КВП).

Исследование КВП на аппарате Нейро-МВП (Нейрософт; Россия), предназначенное для объективизации изменений КФ, выполняли по методике РЗ00. Нормативные данные по потенциалу Р300 были получены у 25 здоровых человек соответствующего возраста. Амплитуду Р300 измеряли от вершины предыдущего негативного пика до вершины пика Р300.

При рассмотрении метода хирургического лечения эндоваскулярное вмешательство выполняли тем 
пациентам, у которых были противопоказания к КЭЭ в соответствии с критериями исследования SAPPHIRE (Stenting and Angioplasty With Protection in Patients at High Risk for Endarterectomy) [17] и предпочтения для KAC в соответствии с критериями исследования CREST [10]. Через 24 ч после открытого хирургического или эндоваскулярного вмешательств проводили неврологическое обследование с оценкой десицита по шкале инсульта National Institutes of Health $(\mathrm{NIH})[18]$.

Состояние вещества головного мозга до вмешательства и через 24 ч после него оценивали по результатам ДВMPT на магнитно-резонансном томографе Magnetom Symphony (Siemens; Германия) с величиной магнитной индукции 1,5 Тесла. Ввиду преобладания очагов малых размеров оценку поражения вещества головного мозга проводили на диффузионно-взвешенных изображениях (с коэффициентом диффузионного взвешивания b 1000) [19].

Всем пациентам не менее чем за 5 дней осуществляли коррекцию антитромботической, гиполипидемической, антигипертензивной и антиангинальной терапией. После операции все пациенты получили базовую медикаментозную терапию (антитромботические, антигипертензивные и гиполипидемические средства). Ноотропные препараты не назначали.

Статистический анализ данных проводили с использованием дисперсионного анализа (с повторными измерениями) и анализа таблиц сопряженности.

\section{РЕЗУЛЬТАТЫ ИССЛЕДОВАНИЯ}

У всех пациентов, включенных в исследование, диагностировали артериальную гипертонию, каждый второй пациент имел два и более факторов риска развития сосудистого заболевания. У 60 (67\%) больных в анамнезе были отмечены перенесенные ишемические нарушения мозгового кровообращения. Анализ исходных данных показал, что нормальные показатели по шкале MMSE (28-30 баллов) были у 33\% пациентов, легкие KH (25-27 баллов) - у 67\% пациентов. По результатам анализа динамики изменения оценок КФ по шкале MMSE через 3, 6 и 9 месяцев после операции, КФ пациентов после операции в целом не ухудшались: к 3 и 6 месяцам были детектированы разнонаправленные изменения КФ (увеличился разброс оценок, появились единичные случаи выраженных изменений КФ), но к 9 месяцам (концу срока наблюдения) эти изменения практически нивелировались и оценки КФ у пациентов приблизились К дооперационным (их различие статистически незначимо; $p=0,43$ ) (рис. 1, 2). Таким образом, наблюдавшиеся послеоперационные изменения КФ носили преимущественно транзиторный характер.

При дополнительном нейропсихологическом тестировании у некоторых пациентов исходно были выявлены умеренные нарушения вербального мышления, внимания и кратковременной памяти (табл. 1). Развитие КН

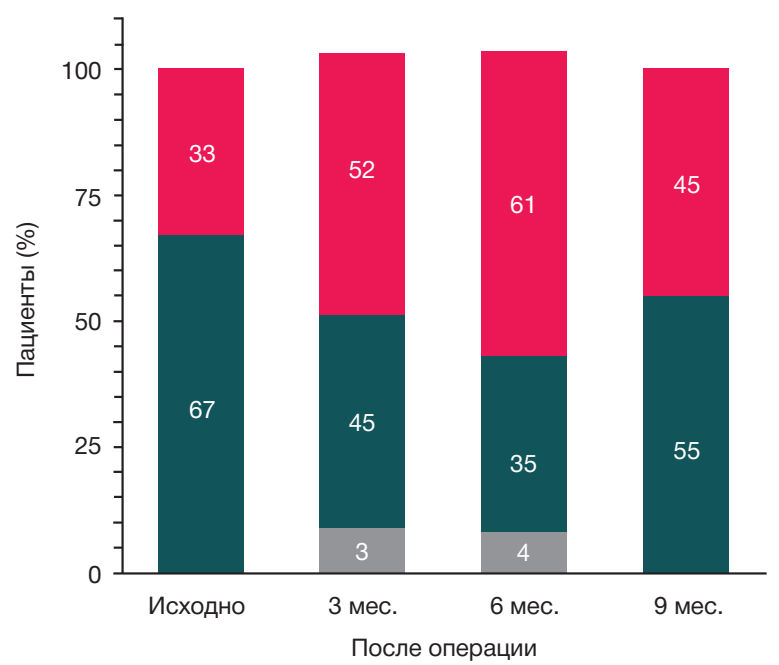

Рис. 1. Динамика изменения соотношения долей пациентов (по оси ординат, в \%) с различными градациями по шкале MMSE: нет KH (28-30 баллов; верхние поля), легкие КН (25-27 баллов; средние поля) и выраженные КН (<25 баллов; два нижних поля)

Таблица 1. Количественная оценка КФ больных (по результатам скрининговых тестов)

\begin{tabular}{|l|c|c|c|c|}
\hline \multirow{2}{*}{ Нейропсихологические тесты } & \multicolumn{4}{|c|}{ Показатели скрининговых тестов } \\
\cline { 2 - 5 } & До лечения & Через 3 месяца & Через 6 месяцев & Через 9 месяцев \\
\hline MMSE & $27,1 \pm 0,2$ & $28,3 \pm 0,1$ & $28,2 \pm 0,1$ & $27,2 \pm 0,1$ \\
\hline БТЛД & $13,1 \pm 0,3$ & $17,4 \pm 0,1$ & $18,1 \pm 0,2$ & $18,7 \pm 0,3$ \\
\hline Проба Шульте (с) & $57,3 \pm 8,5$ & $53,4 \pm 13,1$ & $47,7 \pm 9,6$ & $46,2 \pm 10,3$ \\
\hline Повторение цифр (прямое) & $5,1 \pm 1,1$ & $5,9 \pm 1,1$ & $6,1 \pm 1,1$ & $6,5 \pm 1,2$ \\
\hline Повторение цифр (обратное) & $2,5 \pm 1,1$ & $3,7 \pm 1,0$ & $3,8 \pm 1,1$ & $3,8 \pm 1,1$ \\
\hline Динамический праксис & $1,6 \pm 0,6$ & $2,1 \pm 0,7$ & $2,2 \pm 0,8$ & $2,3 \pm 0,6$ \\
\hline Тест рисования часов & $4,6 \pm 2,2$ & $5,7 \pm 0,2$ & $5,9 \pm 1,1$ & $5,8 \pm 1,0$ \\
\hline Концептуализация & $2,3 \pm 0,7$ & $2,3 \pm 0,1$ & $2,2 \pm 0,4$ & $2,3 \pm 0,3$ \\
\hline
\end{tabular}

Примечание: * - результаты тестов представлены в виде средних значений со стандартными ошибками средних (гистограммы результатов тестов близки к нормальному распределению). 
ассоциировалось с выраженностью атеросклеротического поражения БЦА, пожилым возрастом, наличием артериальной гипертонии, ишемической болезни сердца, сахарного диабета, перенесенным ранее нарушением мозгового кровообращения.

Детальное обследование этих пациентов после оперативного вмешательства выявило, что у одного пациента после КЭЭ развился периоперационный инсульт по типу артерио-артериальной эмболии в бассейне средней мозговой артерии на стороне вмешательства (неврологический десицит по шкале инсульта $\mathrm{NIH}$ достигал 6 баллов), проявившийся в двигательных нарушениях. При дальнейшем наблюдении пациента с периоперационным инсультом и использовании теста вербального мышления и беглости речи было выявлено ухудшение семантической памяти, сохранявшееся на всем протяжении исследования.

Основными нейродинамическими процессами, претерпевшими у пациентов позитивные изменения, были внимание, беглость речи, оперативная и кратковременная память (рис. 3).

Связи вида оперативного вмешательства и оценок КФ у обследованных пациентов не выявлено. Более выраженные КН в течение всего периода наблюдения выявлены у некоторых пациентов старше 60 лет. У большинства пациентов моложе 60 лет (86\%) наблюдали положительные изменения КФ (по шкале MMSE) к 6 месяцу наблюдения, а также увеличивалась доля больных с нормальными показателями при оценке внимания и кратковременной памяти через 9 месяцев (что подтверждали соответствующие изменения амплитуды пика Р300; $p=0,05)$. Влияния ранее перенесенных ишемических инсультов на послеоперационные изменения КФ не обнаружено.

При анализе результатов ДВ-МРТ в ближайшем периоперационном периоде выявлено, что бессимптомные ООИ эмболического генеза визуализировались у 30 (33\%) больных. Выявленные изменения в веществе головного мозга в большинстве случаев были локализованы в корковом веществе (16 (53\%)) на стороне вмешательства (22 (73\%)) и имели размеры до 5 мм. Проведение отдельного анализа данных ДВ-МРТ и КВП показало, что наличие положительной динамики по данным КВП к 3 и 6 месяцам после операции (у пациентов без ООИ) и отсутствие значимой динамики у пациентов с наличием ООИ позволяют рассматривать ООИ как фактор, препятствующий восстановлению КФ после операции (рис. 4).

Небольшая положительная динамика КФ у пациентов без выявленных ООИ в веществе мозга отмечена (через 3, 6 и 9 месяцев) также и при нейропсихологическом тестировании.

Независимых и значимых предикторов, ассоциированных с состоянием пациента через 9 месяцев после оперативного вмешательства, выявлено не было.

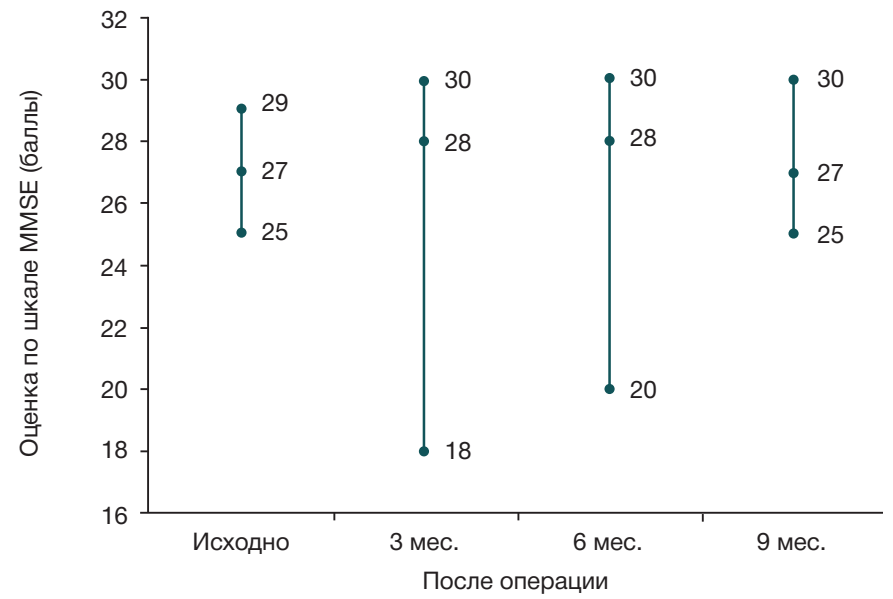

Рис. 2. Динамика изменения оценок КФ (до и в разные сроки после операции) по шкале МMSE: представлены средние величины (с минимальными и максимальными значениями)

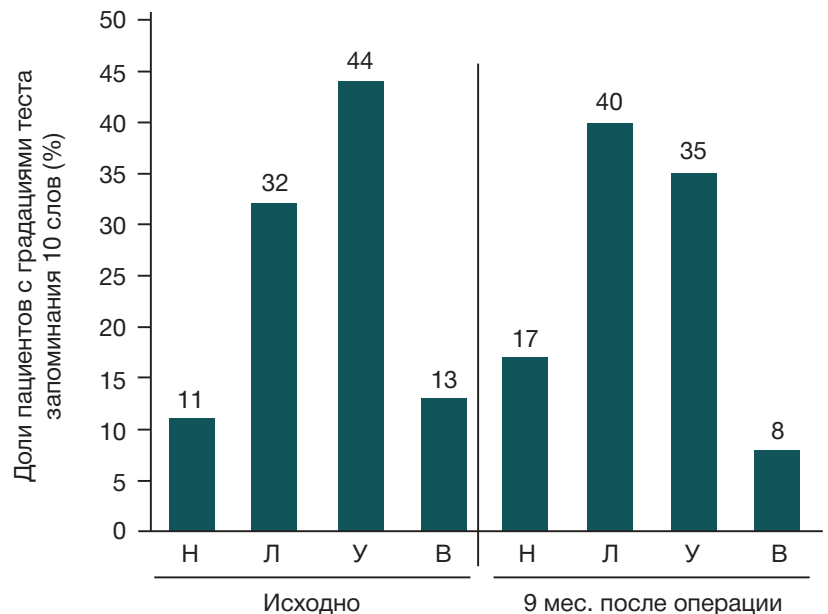

Рис. 3. Сравнение распределений оценок оперативной памяти у пациентов по тесту запоминания 10 слов до операции (исходно) и после операции (к концу срока наблюдения); градации теста: Н — норма (10 слов); Л — легкие нарушения (8-9); У — умеренные (6-7) и В — выраженные (< 6). К концу срока наблюдения (9 месяцам) у пациентов в целом наблюдали улучшение оперативной памяти 
Особую значимость приобретают в контексте изучения КФ данные нейрофизиологического исследования когнитивных вызванных потенциалов Р300. Так, при исходном тестировании 90 пациентов у 22 больных (25\%) характеристики не были изменены, в то время как у 68 больных (75\%) были выявлены следующие отклонения: у 24 отсутствовал пик Р300, у 25 увеличена латентность пика Р300 и у 19 снижена амплитуда. Отметим, что по характеристикам Р300 (приводим средние значения со стандартными отклонениями) у пациентов с артериальной гипертензией и без нее статистически значимых различий не было: 366,4 \pm 29,6 против 360,9 \pm 51,1 мс (латентность) и 5,4 \pm 2,6 против 5,4 \pm 3,2 мкв (амплитуда). По характеристикам Р300 не обнаружено межполушарной асимметрии, а также корреляции количества пораженных экстракраниальных артерий (от одной до четырех).

В послеоперационном периоде средние значения латентности и амплитуды Р300 не отличались от исходных значений. В отдаленном периоде (9 месяцев) у 61\% (55) оперированных больных зарегистрированы уменьшение латентности пика потенциала Р300 и некоторое возрастание амплитуды ответа. Средние значения (со стандартными отклонениями) латентности потенциала P300 до операции - 364,5 \pm 37,5 мс, амплитуды ответа 5,4 \pm 2,7 мкв, после операции - 349,5 \pm 42,7 мс и 6,4 \pm 3,3 мкв соответственно.

\section{ОБСУЖДЕНИЕ РЕЗУЛЬТАТОВ}

Система внутренней сонной артерии обеспечивает около 2/3 кровоснабжения одноименного полушария головного мозга. Атеросклеротическое поражение экстракраниальных артерий существенным образом дискредитирует мозговой кровоток. Гемодинамическое влияние стеноза ВСА на мозговое кровообращение может приводить $\mathrm{K}$ развитию инсульта с явным неврологическим дефицитом или диффузному поражению вещества головного мозга, проявляющемуся негрубой рассеянной неврологической симптоматикой. Изменения КФ после операций исследователей начали интересовать давно. В одном из ранних исследований КФ, касавшемся психопатологических расстройств после кардиальной операции, были отмечены нарушение способности к концентрации внимания, нарушение отсроченной и быстрой памяти, замедленность психомоторных процессов, а факторами риска развития послеоперационных когнитивных осложнений авторы называли старший возраст и гипертонию [20].

С середины 1990-х гг. появились публикации, посвященные изучению КН после операций на сонных артериях. Степень интраоперационной ишемии оценивали путем мониторирования соматосенсорных корковых вызванных потенциалов во время операции. Измененные нейропсихологические показатели в зависимости от степени интраоперационной ишемии были выявлены у пациентов с перенесенным инсультом и с более выраженной степенью поражения артерий [21].

В последующих работах исследовали взаимосвязь между степенью каротидного стеноза и КН у больных без клинических признаков деменции. Показано, что когнитивные функции ухудшаются у недементных больных с высокой степенью стеноза и не меняются при малых степенях. Выявленные мягкие КН по психометрическим тестам коррелировали с изменением латентности потенциала Р300. Измененные потенциалы Р300, связанные с легкими $\mathrm{KH}$, были обнаружены у больных со стенозами BCA (как асимптомных, так и с наличием очаговой симптоматики) [22]. КВП в отличие от психометрических тестов не зависят от мотивации больного.

Учитывая, что результаты некоторых исследований свидетельствуют о значительном улучшении КФ после каротидных вмешательств [23], а в других представлены доказательства их ухудшения [24, 25], вопрос выявления факторов, способствующих изменению когнитивных способностей в результате операции, остается открытым.

В проведенном нами исследовании КФ у пациентов после операций на ВСА выявлено, что в первые 3 месяца разброс оценок КФ (по шкале MMSE) был максимальный, и, возможно, обусловлен влиянием психоэмоционального состояния пациентов в связи с оперативным вмешательством [26]. Основными нейродинамическими процессами, которые претерпевали изменения, были внимание, беглость речи, оперативная и

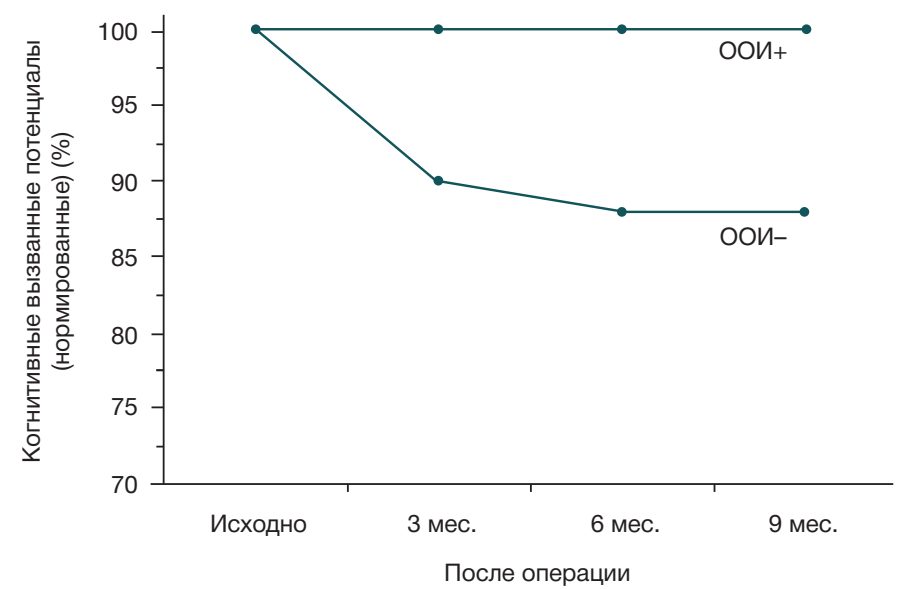

Рис. 4. Динамика изменения КВП в группе пациентов с интраоперационными острыми очагами ишемии (ООИ+) и без них (ООИ-): за период наблюдения уровень КВП в группе ООИ+ $(n=30)$ не изменился, а в группе ООИ- $(n=60)$ снизился на $15 \%(p=0,05)$. Приведены нормированные (деленные на исходные уровни) средние значения КВП (в \%)

Хронические
изменения мозга $\rightarrow \begin{gathered}\text { Острые очаги ишемии } \\ \text { («немые») }\end{gathered} \rightarrow \begin{gathered}\text { Острые очаги ишемии + нарушение } \\ \text { мозгового кровообращения }\end{gathered}$

Рис. 5. Возможные изменения вещества мозга с вектором ухудшения КФ после операции 
кратковременная память. Через 9 месяцев КФ в среднем вернулись к исходному (дооперационному) уровню. Выскажем предположение, что в отдаленные сроки после операции влияние на КФ оказывают исходный статус КФ и адекватность мероприятий в рамках вторичной профилактики сосудистых заболеваний. Кроме этого, вероятными причинами постепенного регресса КН после операции являются восстановление персузии и улучшение метаболизма головного мозга [27, 28].

Результаты нашей работы об отсутствии связи КФ и вида (КЭЭ и КАС) оперативного лечения согласуются с другими исследованиями [29, 30].

Мы выявили (по данным к 6 месяцам наблюдения) связь возраста пациентов (моложе 60 лет) с позитивной динамикой КФ (по шкале MMSE), а также увеличение числа пациентов с нормальными показателями кратковременной памяти и внимания (к 9 месяцам наблюдения), что согласуется с существующими литературными данными [31, 32].

Особенностью нашей работы было сопоставление изменений в веществе головного мозга (по данным ДВMPT) со статусом КФ в различные послеоперационные периоды. Так, в 33\% случаев в ближайшее время после вмешательства были выявлены те или иные ООИ. Вместе с тем, лишь в одном случае нашего наблюдения у пациента развился острый ишемический инсульт с гемипарезом на контрлатеральной стороне, а остальные ООИ были клинически асимптомными

Корреляцию острых ишемических поражений (по данным ДВ-МРТ) и параметров нейропсихологического исхода проводили в ограниченном числе работ [33, 34], в связи с чем сохраняется недостаточность данных по оценке прогностического влияния выявленных поражений на когнитивный статус.

Проведенное нами клиническое нейровизуализационное, нейропсихологическое и нейрофизиологическое исследование позволило сформулировать единую составляющую КФ и установить, что наличие ООИ ухудшает когнитивный прогноз. Динамика этой единой сочетанной меры измерения КФ в остальные сроки наблюдения зависит от их клинического проявления. В связи с этим можно предложить вероятный прогноз ухудшения КФ после операции по поводу атеросклеротического стеноза сонной артерии (рис. 5).

Ограничением исследования является сравнительно короткий срок наблюдения (9 месяцев). Продолжение исследования позволит верифицировать полученные результаты.

\section{ВЫВОДЫ}

1. Одним из важнейших симптомов хронических цереброваскулярных заболеваний у большинства пациентов с атеросклеротической патологией сонных артерий является когнитивная диссункция, требующая при планировании ангиореконструктивных операций обязательного нейропсихологического, нейровизуализационного и нейрофизиологического тестирования. 2. Когнитивные нарушения у пациентов после ангиореконструктивных операций на сонных артериях сопряжены с возможными изменениями (симптомными или асимптомными) состояния вещества головного мозга.

\section{Литература}

1. Танашян М. М., Медведев Р. Б., Евдокименко А. Н., Гемджян Э. Г., Скрылев С. И., Лагода О. В., и др. Прогнозирование ишемических повреждений головного мозга при реконструктивных операциях на внутренних сонных артериях. Ангиология и сосудистая хирургия. 2017; 23 (1): 59-65.

2. Bossema ER, Brand N, Moll FL, Ackerstaff RG, de Haan EH, van Doornen LJ. Cognitive functions in carotid artery disease before endarterectomy. J Clin Exp Neuropsychol. 2006; (28): 357-69.

3. Johnston SC, O'Meara ES, Manolio TA, Lefkowitz D, O'Leary DH, Goldstein S, et al. Cognitive impairment and decline are associated with carotid artery disease in patients without clinically evident cerebrovascular disease. Ann Intern Med. 2004;140 (4): 237-47.

4. Romero JR, Beiser A, Seshadri S, Benjamin EJ, Polak JF, Vasan RS, et al. Carotid artery atherosclerosis, MRI indices of brain ischemia, aging, and cognitive impairment: the Framingham study. Stroke. 2009; 40 (5): 1590-6.

5. Кунцевич Г. И., Танашян М. М., Скрылев С. И., Кротенкова М. В., Щипакин В. Л., Кощеев А. Ю., и др. Интраоперационное мониторирование мозгового кровотока и состояние вещества головного мозга при открытых и эндоваскулярных вмешательствах в каротидной системе. Ангиология и сосудистая хирургия. 2011; 17 (3): 43-8.

6. Barnett HJM, Taylor DW, Haynes RB, Sackett DL, Peerless SJ, Ferguson GG, et al. North American Symptomatic Carotid Endarterectomy Trial Collaborators. Beneficial effect of carotid endarterectomy in symptomatic patients with high grade carotid stenosis. N Engl J Med. 1991; 325 (7): 445-53.

7. Endarterectomy for asymptomatic carotid artery stenosis. Executive Committee for the Asymptomatic Carotid Atherosclerosis Study. JAMA. 1995; 273 (18): 1421

8. Endovascular versus surgical treatment in patients with carotid

stenosis in the Carotid and Vertebral Artery Transluminal Angioplasty Study (CAVATAS): a randomised trial. Lancet. 2001: 357 (9270): 1729-37.

9. Mas JL, Trinquart L, Leys D, Albucher JF, Rousseau H, Viguier A, et al. Endarterectomy Versus Angioplasty in Patients with Symptomatic Severe Carotid Stenosis (EVA-3S) trial: results up to 4 years from a randomised, multicentre trial. Lancet Neurol. 2008; 7 (10): 885-92.

10. Brott TG, Hobson RW, Howard G, Roubin GS, Clark WM, Brooks W, et al. Stenting versus endarterectomy for treatment of carotidartery stenosis. N Engl J Med. 2010; 363 (1): 11-23.

11. Медведев Р. Б., Танашян М. М., Скрылев С. И., Гемджян Э. Г., Гулевская Т. С., Ануфриев П. Л. Связь ультразвуковых и морфологических характеристик атеросклеротических бляшек каротидного синуса. Ангиология и сосудистая хирургия. 2018; 24 (4): 43-9.

12. Altinbas $A$, van Zandvoort $M J$, van den Berg $E$, Jongen $L M$, Algra A, Moll FL, et al. Cognition after carotid endarterectomy or stenting a randomized comparison. Neurology. 2011; 77 (11): 1084-90.

13. Zhou W, Hitchner E, Gillis K, Sun L, Floyd R, Lane B, et al. Prospective neurocognitive evaluation of patients undergoing carotid interventions. J Vasc Surg. 2012; 56 (6): 1571-8.

14. Barnett HJ, Taylor DW, Eliasziw M, Fox AJ, Ferguson GG, Haynes RB, et al. Benefit of carotid endarterectomy in patients with symptomatic moderate or severe stenosis. North American Symptomatic Carotid Endarterectomy Trial Collaborators. N Engl J Med. 1998; 339 (20): 1415-25.

15. Folstein MF, Folstein SE, McHugh PR. "Mini-mental state". A practical method for grading the cognitive state of patients for the clinician. J Psychiatr Res. 1975; 12 (3): 189-98.

16. Яхно Н. Н. Когнитивные нарушения в неврологической практике. Неврологический журнал. 2006; 11 (1): 4-12. 
17. Yadav JS, Wholey MH, Kuntz RE, Fayad P, Katzen BT, Mishkel GJ, et al. Protected carotid-artery stenting versus endarterectomy in high-risk patients. N Engl J Med. 2004; 351 (15): 1493-501.

18. Brott T, Adams HP Jr, Olinger CP, Marler JR, Barsan WG, Biller J, et al. Measurements of acute cerebral infarction: a clinical examination scale. Stroke. 1989; 20 (7): 864-70.

19. Медведев Р. Б., Танашян М. М., Кунцевич Г. И., Лагода О. В., Скрылев С. И., Кротенкова М. В., и др. Ишемические повреждения головного мозга после каротидного стентирования. Ангиология и сосудистая хирургия. 2015; 21 (1): $65-71$.

20. Fox HM, Rizzo ND, Gifford S. Psychological observations of patients undergoing mitral surgery; a study of stress. Psychosom Med. 1954; 16 (3): 186-208.

21. Kügler $C F$, Vlajic $P$, Funk $H$, Raithel $D$, Platt $D$, et al. The event-related P300 potential approach to cognitive functions of nondemented patients with cerebral and peripheral arteriosclerosis. J Am Geriatr Soc. 1995; 43 (11): 1228-36.

22. Inoue T, Ohwaki K, Tamura A, Tsutsumi K, Saito I, Saito N. Subclinical ischemia verified by somatosensory evoked potential amplitude reduction during carotid endarterectomy: negative effects on cognitive performance clinical article. J Neurosurg. 2013; 118 (5): 1023-9.

23. Xu G, Liu X, Meyer JS, Yin Q, Zhang R. Cognitive performance after carotid angioplasty and stenting with brain protection devices. Neurol Res. 2007; (29): 251-55.

24. Witt K, Borsch K, Daniels C, Walluscheck K, Alfke K. Neuropsychological consequences of endarterectomy and endovascular angioplasty with stent placement for treatment of symptomatic carotid stenosis: a prospective randomised study. $J$ Neurol. 2007; (254): 1524-32.

25. Lal BK, Younes M, Cruz G, Kapadia I, Jamil Z, Pappas PJ. Cognitive changes after surgery vs stenting for carotid artery stenosis. J Vasc Surg. 2011; 54 (3): 691-8.

26. Выборных Д. Э., Федорова С. Ю., Хрущев С. О., Дроков М. Ю., Гемджян Э. Г., Кузьмина Л.А., и др. Когнитивные нарушения у пациентов с заболеваниями системы крови, перенесших трансплантацию аллогенных гемопоэтических стволовых клеток. Обозрение психиатрии и медицинской психологии им. В. М. Бехтерева. 2019; (2): 18-26.

27. Shimada Y, Kobayashi M, Yoshida K, Terasaki K, Fujiwara S, Kubo Y, et al. Reduced Hypoxic Tissue and Cognitive Improvement after Revascularization Surgery for Chronic Cerebral Ischemia. Cerebrovasc Dis. 2019; 47 (1-2): 57-64.

28. List J, Hertel-Zens S, Kübke JC, Lesemann A, Schreiber SJ, Flöel A. Cortical reorganization due to impaired cerebral autoregulation in individuals with occlusive processes of the internal carotid artery. Brain Stimul. 2014; 7 (3): 381-7.

29. Kuo-Lun Huang, Ting-Yu Chang, Meng-Yang Ho, Wei-Hao Chen, Mei-Yu Yeh, Yeu-JhyChang, et al. The correlation of asymmetrical functional connectivity with cognition and reperfusion in carotid stenosis patients. Neuro Image Clinical. 2018; (20): 476-84.

30. Crawley F, Stygall J, Lunn S, Harrison M, Brown MM. Comparison of microembolism detected by transcranial Doppler and neuropsychological sequelae of carotid surgery and percutaneous transluminal angioplasty. Stroke. 2000; (31): 1329-34.

31. Ortega G, Alvarez B, Quintana M, Yugueros X, Alvarez-Sabin J, Matas M. Asymptomatic carotid stenosis and cognitive improvement using transcervical stenting with protective flow reversal technique. Eur J Vasc Endovasc Surg. 2014; 47 (6): 585-92

32. Turan TN, Smock A, Cotsonis G, Bachman D, Al Kasab S, Lynn MJ, et al. SAMMPRIS Investigators. Is There Benefit from Stenting on Cognitive Function in Intracranial Atherosclerosis? Cerebrovasc Dis. 2017; 43 (1-2): 31-5.

33. Танашян М. М., Медведев Р. Б., Гемджян Э. Г., Скрылев С. И. Кротенкова М. В., Щипакин В. Л., и др. Предикторь острых церебральных эмболических повреждений при стентировании сонной артерии. Ангиология и сосудистая хирургия. 2019; 25 (4). (В печати)

34. Barber PA, Hach S, Tippett LJ, Ross L, Merry AF. Cerebral ischemic lesions on diffusion-weighted imaging are associated with neurocognitive decline after cardiac surgery. Stroke. 2008; (39): 1427-33.

\section{References}

1. Tanashyan MM, Medvedev RB, Evdokimenko AN, Gemdzhian EG, Skrylev SI, Lagoda OV, et al. Prediction of ischaemic lesions of the brain in reconstructive operations on internal carotid arteries. Angiol Sosud Khir. 2017; 23 (1): 59-65.

2. Bossema ER, Brand N, Moll FL, Ackerstaff RG, de Haan EH, van Doornen LJ. Cognitive functions in carotid artery disease before endarterectomy. J Clin Exp Neuropsychol. 2006; (28): 357-69.

3. Johnston SC, O'Meara ES, Manolio TA, Lefkowitz D, O'Leary DH, Goldstein S, et al. Cognitive impairment and decline are associated with carotid artery disease in patients without clinically evident cerebrovascular disease. Ann Intern Med. 2004; 140 (4): 237-47.

4. Romero JR, Beiser A, Seshadri S, Benjamin EJ, Polak JF, Vasan RS, et al. Carotid artery atherosclerosis, MRI indices of brain ischemia, aging, and cognitive impairment: the Framingham study. Stroke. 2009; 40 (5): 1590-6.

5. Kuntsevich GI, Tanashyan MM, Skrylev SI, Krotenkova MV, Shchipakin VL, Koshcheev Alu, et al. Intraoperative monitoring of cerebral blood-flow and condition of cerebral at open and endovascular interventions in carotid system. Angiol Sosud Khir 2011; 17 (3): 43-8.

6. Barnett HJM, Taylor DW, Haynes RB, Sackett DL, Peerless SJ, Ferguson GG, et al. North American Symptomatic Carotid Endarterectomy Trial Collaborators. Beneficial effect of carotid endarterectomy in symptomatic patients with high grade carotid stenosis. N Engl J Med. 1991; 325 (7): 445-53.

7. Endarterectomy for asymptomatic carotid artery stenosis. Executive Committee for the Asymptomatic Carotid Atherosclerosis Study. JAMA. 1995; 273 (18): 1421.

8. Endovascular versus surgical treatment in patients with carotid stenosis in the Carotid and Vertebral Artery Translumina Angioplasty Study (CAVATAS): a randomised trial. Lancet. 2001;

357 (9270): 1729-37

9. Mas JL, Trinquart L, Leys D, Albucher JF, Rousseau H, Viguier A, et al Endarterectomy Versus Angioplasty in Patients with Symptomatic Severe Carotid Stenosis (EVA-3S) trial: results up to 4 years from a randomised, multicentre trial. Lancet Neurol. 2008; 7 (10): 885-92.

10. Brott TG, Hobson RW, Howard G, Roubin GS, Clark WM, Brooks W, et al. Stenting versus endarterectomy for treatment of carotid-artery stenosis. N Engl J Med. 2010; 363 (1): 11-23

11. Medvedev RB, Tanashyan MM, Skrylev SI, Gemdzhian EG, Gulevskaya TS, Anufriev PL. Relation between ultrasonographic and morphological characteristics of atherosclerotic plaques of carotid sinus. Angiol Sosud Khir. 2018; 24 (4): 43-9.

12. Altinbas $A$, van Zandvoort $M J$, van den Berg $E$, Jongen $L M$, Algra A, Moll FL, et al. Cognition after carotid endarterectomy or stenting a randomized comparison. Neurology. 2011; 77 (11): 1084-90.

13. Zhou W, Hitchner E, Gillis K, Sun L, Floyd R, Lane B, et al. Prospective neurocognitive evaluation of patients undergoing carotid interventions. J Vasc Surg. 2012; 56 (6): 1571-8.

14. Barnett HJ, Taylor DW, Eliasziw M, Fox AJ, Ferguson GG, Haynes RB, et al. Benefit of carotid endarterectomy in patients with symptomatic moderate or severe stenosis. North American Symptomatic Carotid Endarterectomy Trial Collaborators. N Engl J Med. 1998; 339 (20): 1415-25.

15. Folstein MF, Folstein SE, McHugh PR. "Mini-mental state". A practical method for grading the cognitive state of patients for the clinician. J Psychiatr Res. 1975; 12 (3): 189-98.

16. Yakhno NN. Cognitive impairment in neurological practice. Nevrologicheskii Zhurnal. 2006; 11 (1): 4-12.

17. Yadav JS, Wholey MH, Kuntz RE, Fayad P, Katzen BT, Mishkel GJ, et al. Protected carotid-artery stenting versus endarterectomy in 
high-risk patients. N Engl J Med. 2004; 351 (15): 1493-501.

18. Brott T, Adams HP Jr, Olinger CP, Marler JR, Barsan WG, Biller J, et al. Measurements of acute cerebral infarction: a clinical examination scale. Stroke. 1989; 20 (7): 864-70.

19. Medvedev RB, Tanashian MM, Kuntsevich Gl, Lagoda OV, Skrylev SI, Krotenkova MV, et al. Ischaemic lesions of cerebral after carotid stenting. Angiol Sosud Khir. 2015; 21 (1): 65-71.

20. Fox HM, Rizzo ND, Gifford S. Psychological observations of patients undergoing mitral surgery; a study of stress. Psychosom Med. 1954; 16 (3): 186-208.

21. Kügler CF, Vlajic P, Funk H, Raithel D, Platt D, et al. The event-related P300 potential approach to cognitive functions of nondemented patients with cerebral and peripheral arteriosclerosis. J Am Geriatr Soc. 1995; 43 (11): 1228-36.

22. Inoue T, Ohwaki K, Tamura A, Tsutsumi K, Saito I, Saito N. Subclinical ischemia verified by somatosensory evoked potential amplitude reduction during carotid endarterectomy: negative effects on cognitive performance clinical article. J Neurosurg. 2013; 118 (5): 1023-9.

23. Xu G, Liu X, Meyer JS, Yin Q, Zhang R. Cognitive performance after carotid angioplasty and stenting with brain protection devices. Neurol Res. 2007; (29): 251-55.

24. Witt K, Borsch K, Daniels C, Walluscheck K, Alfke K. Neuropsychological consequences of endarterectomy and endovascular angioplasty with stent placement for treatment of symptomatic carotid stenosis: a prospective randomised study. $J$ Neurol. 2007; (254): 1524-32.

25. Lal BK, Younes M, Cruz G, Kapadia I, Jamil Z, Pappas PJ. Cognitive changes after surgery vs stenting for carotid artery stenosis. J Vasc Surg. 2011; 54 (3): 691-8.

26. Vybornykh DE, Fedorova SYu, Khrushchev SO, Drokov MYu, Gemdzhian EG, Kuzmina LA, Parovichnikova EN. Cognitive impairments in patients with hematological malignancies prior and after allogeneic hematopoietic stem cells transplantation.
Obozrenie psihiatrii i medicinskoj psihologii im VM Behtereva. 2019; (2): 18-26.

27. Shimada Y, Kobayashi M, Yoshida K, Terasaki K, Fujiwara S, Kubo Y, et al. Reduced Hypoxic Tissue and Cognitive Improvement after Revascularization Surgery for Chronic Cerebral Ischemia. Cerebrovasc Dis. 2019; 47 (1-2): 57-64.

28. List J, Hertel-Zens S, Kübke JC, Lesemann A, Schreiber SJ, Flöel A. Cortical reorganization due to impaired cerebral autoregulation in individuals with occlusive processes of the internal carotid artery. Brain Stimul. 2014; 7 (3): 381-7.

29. Kuo-Lun Huang, Ting-Yu Chang, Meng-Yang Ho, Wei-Hao Chen, Mei-Yu Yeh, Yeu-JhyChang, et al. The correlation of asymmetrical functional connectivity with cognition and reperfusion in carotid stenosis patients. Neuro Image Clinical. 2018; (20): 476-84.

30. Crawley F, Stygall J, Lunn S, Harrison M, Brown MM. Comparison of microembolism detected by transcranial Doppler and neuropsychological sequelae of carotid surgery and percutaneous transluminal angioplasty. Stroke. 2000; (31): 1329-34.

31. Ortega G, Alvarez B, Quintana M, Yugueros X, Alvarez-Sabin J, Matas M. Asymptomatic carotid stenosis and cognitive improvement using transcervical stenting with protective flow reversal technique. Eur J Vasc Endovasc Surg. 2014; 47 (6): 585-92.

32. Turan TN, Smock A, Cotsonis G, Bachman D, Al Kasab S, Lynn MJ, et al. SAMMPRIS Investigators. Is There Benefit from Stenting on Cognitive Function in Intracranial Atherosclerosis? Cerebrovasc Dis. 2017; 43 (1-2): 31-5.

33. Tanashyan MM, Medvedev RB, Gemdzhian EG, Skrylev SI, Krotenkova MV, Shchipakin VL, et al. Predictors of acute cerebral embolic lesions during carotid artery stenting. Angiol Sosud Khir. 2019; 25 (4). (In Print)

34. Barber PA, Hach S, Tippett LJ, Ross L, Merry AF. Cerebral ischemic lesions on diffusion-weighted imaging are associated with neurocognitive decline after cardiac surgery. Stroke. 2008; (39): 1427-33. 